Gentle and fast atomic force microscopy with a piezoelectric scanning probe for nanorobotics applications

This article has been downloaded from IOPscience. Please scroll down to see the full text article.

2013 Nanotechnology 24065502

(http://iopscience.iop.org/0957-4484/24/6/065502)

View the table of contents for this issue, or go to the journal homepage for more

Download details:

IP Address: 86.195.7.172

The article was downloaded on 22/01/2013 at 21:30

Please note that terms and conditions apply. 


\title{
Gentle and fast atomic force microscopy with a piezoelectric scanning probe for nanorobotics applications
}

\author{
Juan Camilo Acosta ${ }^{1}$, Jérôme Polesel-Maris ${ }^{3}$, François Thoyer $^{3}$, \\ Hui Xie $^{4}$, Sinan Haliyo ${ }^{2}$ and Stéphane Régnier ${ }^{2}$ \\ ${ }^{1}$ Departamento de Automática y Electrónica, Universidad Autónoma de Occidente, Cll 25 N 115-85 \\ Km. 2 Vía Cali-Jamundí, Colombia \\ ${ }^{2}$ Institut des Systèmes Intelligents et de Robotique, Université Pierre et Marie Curie, CNRS UMR 7222, \\ 4 Place Jussieu, F-75252 Paris Cedex, France \\ ${ }^{3}$ CEA, IRAMIS, Service de Physique et Chimie des Surfaces et Interfaces, F-91191 Gif-sur-Yvette, \\ France \\ ${ }^{4}$ The State Key Laboratory of Robotics and Systems, Harbin Institute of Technology, 2 Yikuang Str., \\ 150080 Harbin, People's Republic of China
}

E-mail: jcacosta@uao.edu.co and jerome.polesel@cea.fr

Received 23 July 2012, in final form 10 November 2012

Published 22 January 2013

Online at stacks.iop.org/Nano/24/065502

\begin{abstract}
A novel dual tip nanomanipulation atomic force microscope (AFM) platform operating in ambient conditions is presented. The system is equipped with a high frequency quartz piezoelectric self-sensing scanning probe for fast imaging and a passive cantilever for manipulation. The system is validated by imaging and selective pushing/pulling of gold colloid beads (diameters from 80 to $180 \mathrm{~nm}$ ). This provides a more compact integration compared to an external optical lever and avoids several of its drawbacks such as optical interference and noise, and recalibration in the case of a moving cantilever and a fixed laser source and photodiode sensor. Moreover, as the quartz oscillator exhibits oscillation amplitudes in the sub-picometer range with a resonant frequency in the megahertz range, this dynamic force sensor is ideal for fast AFM imaging. Experiments show an increase by five times in imaging speed compared to a classical AFM system.
\end{abstract}

(Some figures may appear in colour only in the online journal)

\section{Introduction}

New tools for the assembly of MEMSs and NEMSs (micro/nano electro-mechanical systems) in efficient and repeatable ways are required today for novel applications of nanotechnologies [1-3]. New strategies for fast micro- and nanomanipulation are in demand for the fabrication of new proof-of-concept nanosystems in laboratories, but also for industrial production. Micro/nanoassembly in high vacuum conditions of SEM (scanning electron microscopy) appears to be a promising approach; nevertheless, it remains very expensive due to the equipment cost and it is unpractical for industrial applications because of the requirement of confinement of the SEM chamber [4]. The development of micro/nanoassembly techniques in ambient conditions could hence remove most of these constraints and generalize these assembly, characterization and fabrication processes [5].

Micromanipulation in ambient conditions is generally performed by microtweezers or atomic force microscopes (AFMs) [6-10]. The former have mainly been used for picking and placing of micro-objects of more than $10 \mu \mathrm{m}$ [3]. Their lack of dexterity and force feedback, the adhesion effects between the tool and the object, and their dependence on an external visual sensing device are the primary factors that limit their widespread use for smaller objects. On the other hand, AFMs, compared to traditional microtweezers, 


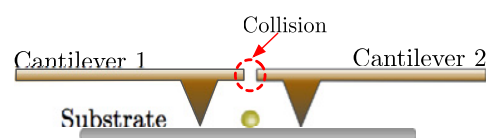

(a)

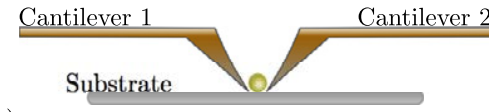

(b)

Figure 1. Tip-object interaction with a two-tip AFM. (a) Approach with a classical AFM cantilever, where cantilever collision is possible, (b) use of protruding tips to avoid kinematical constraints during approach and (c) an AFM with a cantilever with a protruding tip and a quartz oscillator. The length of the tip attached to the oscillator avoids contact between the cantilever and the body of the quartz sensor.

can sense forces in a wider range, from tens of $\mathrm{pN}$ up to the $\mu \mathrm{N}$ range. This sensitivity to low forces in addition to their ability to image the surface removes the dependence on external visual sensing and allows reversible manipulation of smooth and fragile objects. AFMs have been widely used for 2D micro- and nanomanipulation in the air by pushing/pulling procedures [11-14]. One of the drawbacks of $2 \mathrm{D}$ nanomanipulation with traditional AFMs is that the same tip is used for imaging and manipulation. Hence, these two procedures need to be accomplished serially. Recently, out-of-plane spatial manipulation has been achieved by building microtweezers with two independently controlled AFM arms with optical levers [5]. This greatly improves the potential use of the AFM by increasing the capabilities and efficiency of the manipulation process for 3D microand nanomanipulation in ambient conditions. Furthermore, one of the tips can be used for imaging while the other is used for manipulation, making 2D manipulation in parallel with imaging possible [15]. This application of the two-tip AFM, like the classical AFM, remains limited by the speed of image acquisition. Recent progress seems to confirm that some improvements are possible [16-20]. Additionally, it is limited in dexterity because of the kinematic complexity of the setup: as the photodiode sensor and laser source are fixed with respect to a moving cantilever, the force acquisition is spatially limited to a small range.

In this work, a new approach to improve the imaging speed of an AFM system and its dexterity is proposed, by replacing the AFM probe with a faster and self-sensing one, exploring in this way the dynamics of the nanoworld. Dynamic force sensors working in amplitude modulation (AM-AFM), or frequency modulation (FM-AFM) with small amplitudes of oscillation (less than a few nanometers) allow minimization of the contact operation on the sample, which limits the shear forces applied to the surfaces of objects. This allows the exploration of soft and fragile materials, even with stiff probes such as quartz sensors [21]. Quartz piezoelectric tuning fork (TF) probes are proposed for true-non-contact mode imaging $[22,2]$ with small amplitudes of oscillation and for nanocharacterization [23] by directly reading the mechanical properties of the nanostructure with gradient force sensing estimated with frequency modulation [24]. Tuning forks can be found with resonant frequencies of up to $200 \mathrm{kHz}$ [25]. The low resonant frequency directly affects the force feedback rate, thus limiting the imaging speed in force microscopy to tens of minutes. In this work, a novel dynamic force sensor with resonant frequencies in the range of a few megahertz for high speed parallel imaging and manipulation is proposed. This frequency range for the scanning probe would allow the achievement of tens of millisecond frame rates for AFM imaging with an optimized scanner, as demonstrated with optical lever detection cantilevers [19]. An AFM system with two probes is proposed here, with one AFM cantilever for manipulation and one self-sensing scanning probe for fast noninvasive AFM image scanning. In section 2, the manipulation setup using the proposed new probe will be presented. The dynamics and sensitivity of the sensor are then analyzed, followed by imaging and combined imaging and manipulation experiments on nanobeads.

\section{Description of the experimental setup}

\subsection{The manipulation system}

Classical AFM cantilevers have a tip attached/edged at a $90^{\circ}$ angle with the lever. Additionally, they are positioned far from the end of the lever. In consequence, approaching the tips of the two AFM cantilevers is challenging due to the geometrical constraints (figure 1(a)). This issue has been solved by using AFM cantilevers with protruding tips, as seen in figure 1(b) $[5,15,26]$. In this work, a two-tip system based on one AFM cantilever with a protruding tip and one quartz oscillator is proposed. The protruding tip of the cantilever combined with the length of the tip attached to the quartz makes collision between the bodies of the two sensors unlikely, and collision between the two tips possible (figure 1(c)).

One of the advantages of this system is that the kinematical configuration of the piezoactuators as well as the force sensors can be easily modified and adapted to specific applications. The system diagram of the new system is represented in figure 2 and can be described as follows.

(i) The AFM cantilever (called tip I) is mounted on an $\mathrm{X}-\mathrm{Y}-\mathrm{Z}$ micro-positioning stage for coarse positioning; it remains fixed during the manipulation process.

(ii) The quartz probe for AFM image scanning (called tip II) is mounted on an $X-Y-Z$ piezoscanner (Physik 


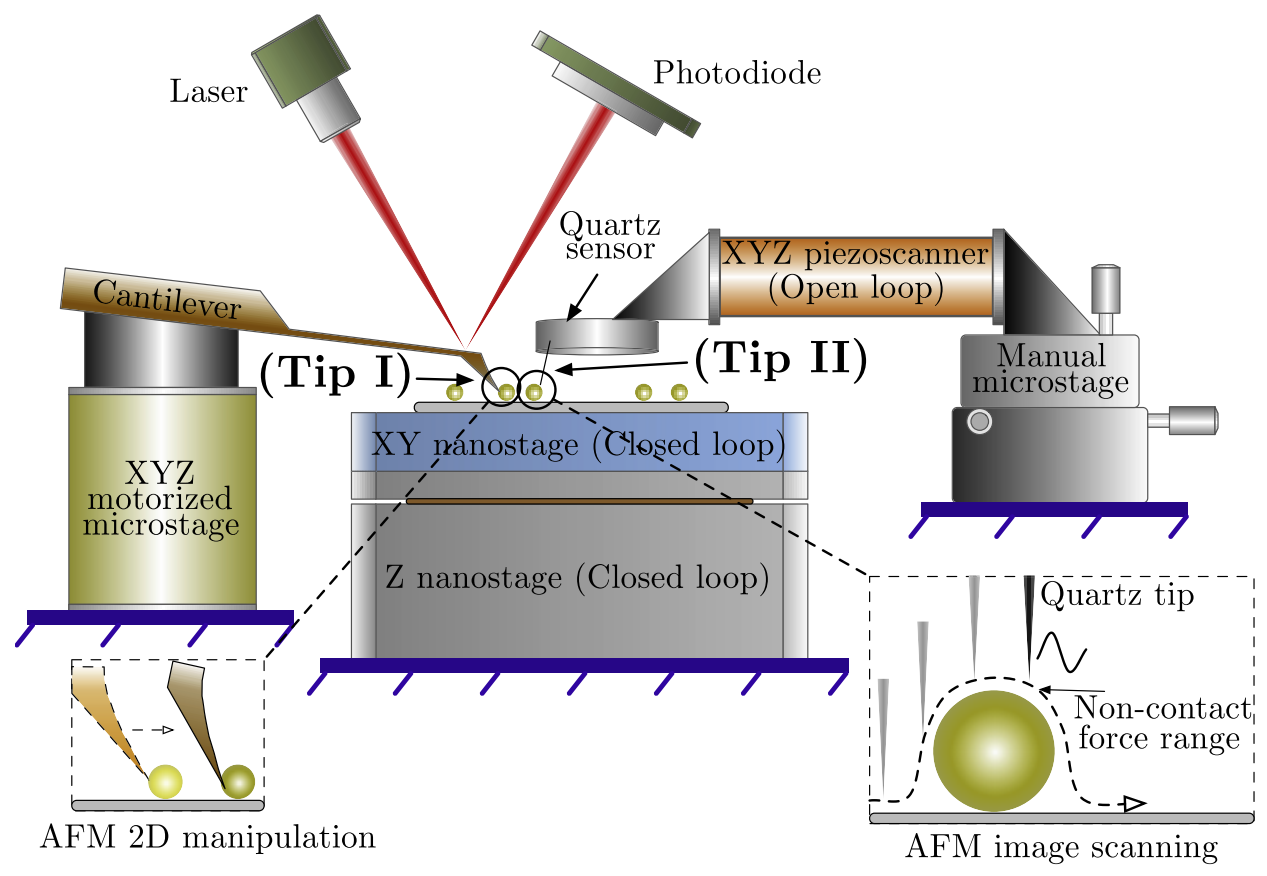

Figure 2. System diagram for the new configuration of the two-tip system. The quartz is integrated into the system for true-non-contact-mode imaging.

Instruments, $\mathrm{p}-153.10 . \mathrm{H})$ with a motion range of $10 \mu \mathrm{m}$ and a sub-nanometer resolution on each axis. The piezotube is well compensated by the Prandtl-Ishlinskii operator on its hysteresis [27] and is mounted on an $X-Y-Z$ manual microstage. For the oscillation control of the quartz oscillator, an OC4-Station from SPECS-Nanonis (Zürich, Switzerland) is used. This station has the advantage of integrating all the systems for fast dynamic AFM operation, until $5 \mathrm{MHz}$ of bandwidth: a lock-in amplifier, a phase locked loop (PLL), an automatic gain control (AGC), data acquisition hardware and software and a real time operating system. A custom home-built transimpedance preamplifier to drive the high frequency piezoelectric scanning probe has been built to develop a gain of $G_{\text {trans }}=6.37 \mathrm{M} \Omega$ over $4.6 \mathrm{MHz}$ of bandwidth, as illustrated in figure 3 . The noise level of detection has been measured to be $1.57 \mathrm{pA} \mathrm{Hz}^{-1 / 2}$.

(iii) An $X-Y-Z$ nanostage (MCL Nano-Bio2M on the $X-Y$ axes, PI P-732.ZC on the $Z$-axis) with a maximum motion range of $50 \mu \mathrm{m} \times 50 \mu \mathrm{m} \times 10 \mu \mathrm{m}$ and a closed loop sub-nanometer resolution is used to drive the sample.

(iv) Two data acquisition cards (National Instruments NI6289 and NI6259) with resolutions of 18 and 16 bits in A/D transfer and maximum sampling frequencies of $625 \mathrm{kHz}$ and $1.25 \mathrm{MHz}$ are used for data acquisition from the optical lever, the amplifier of the cantilever and the transimpedance preamplifier of the high frequency quartz scanning probe, and to actuate both of the $X-Y-Z$ piezotubes by outputting voltage signals to three independent high voltage amplifiers.

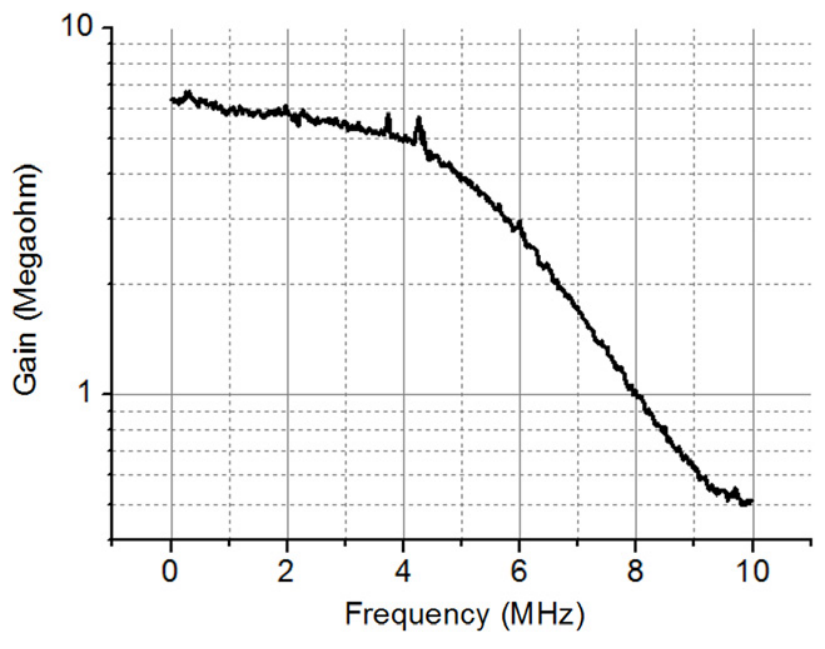

Figure 3. Experimental characterization of the gain and the bandwidth of the custom home-built transimpedance preamplifier to drive the high frequency piezoelectric scanning probes.

AFM imaging with the quartz oscillator tip (tip II) can be carried out by moving the substrate with the closed loop stage or by moving the sensor with the open loop piezoscanner. The latter has the advantage of avoiding movement of the substrate, thus keeping the distance between the cantilever tip (tip I), and substrate constant during imaging. The closed loop nanostage and tip I are used for manipulation.

\subsection{Force sensor preparation}

Dynamic force sensing is an alternative for increasing the speed and the dexterity of the system. This technique has 


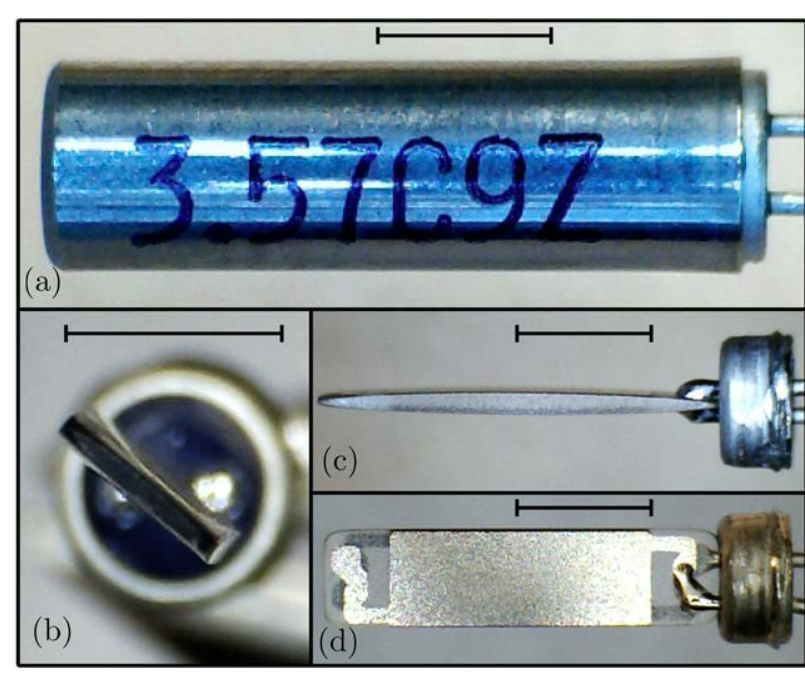

Figure 4. Different views of the quartz sensor. The scale bar is $2.7 \mathrm{~mm}$. (a) The original packaging of the quartz, (b) top view and (c)-(d) side views.

generally been used with quartz tuning forks with a typical resonance frequency of $32.768 \mathrm{kHz}$ [2]. For high speed AFM, even using frequency modulation, these sensors remain slow due to their low data rate. In consequence, in order to take advantage of dynamic force sensing for AFM imaging, the use of quartz oscillators with higher resonance frequencies of several megahertz is preferable. The quartz resonators used (Citizen America CSA310) have a resonant frequency of $3.579545 \mathrm{MHz}$ according to the manufacturer. The geometry of this quartz is different with a monolithic rectangular and convex AT-cut quartz body [28] compared to the two-prong geometry of a tuning fork. The resonance frequency, and also the spring constant of this resonator for shear wave oscillations, is mainly determined by the material properties and the thickness of the crystal, as explained in detail in [28]. Figure 4 shows different views of the oscillator. The packaging (figure 4(a)) is very similar to a tuning fork: the quartz is isolated from the outside by a capsule in vacuum. This capsule is mechanically removed by tweezers to transform the high frequency resonator into a scanning probe. Then, to obtain the AFM probe, a tungsten tip (T-4-10-1 mm, tip radius $100 \mathrm{~nm}$, GGB Industries) is glued to the resonator using the electrically conductive silver epoxy Epotek H21D (Epoxy Technology). The long tip (millimeter range) configuration of the stiff monolithic quartz probe marks another radical difference compared to a standard AFM using silicon based cantilevers with a micrometer range tip. This needle-like configuration paves the way to new opportunities for sample access of the scanning probe, where the embodiment of a standard AFM cantilever can hinder nanoscale imaging or mechanical characterization as its tip is too short.

The quality factors of several scanning probes have been characterized as between 10000 and 200000, depending on the position of the tip on the monolith and the weight of glue added. An example of frequency sweep can be seen in figure 5. For this quartz with a tip, the resonance frequency is measured as $f_{0}=3.57910104 \mathrm{MHz}$ and the quality factor is $Q=125 k$. The stability of the OC4 PLL controller (SPECS-Nanonis) is given as $1 \mathrm{ppb} \mathrm{d}^{-1}$, but with a noise figure in the frequency, considering the probe and our preamplifier, this limits the precision for tracking the resonance frequency shift during scanning to $10 \mathrm{MHz}$. The mechanical amplitude of oscillation for the surface oscillation mode of the quartz tip is estimated as $A=166 \mathrm{fm}$ with [29, 30]

$$
A=\frac{V_{\text {out }}}{G_{\text {trans }}} \frac{1}{2 \pi f_{0} K d},
$$

where $V_{\text {out }}$ and $G_{\text {trans }}$ are the output voltage and the gain of the transimpedance preamplifier respectively, $f_{0}$ and $K$ are the frequency and the spring constant of the oscillation mode of the quartz resonator respectively and $d$ is the piezoelectric coefficient of the piezoelectric strain coefficient, which is equal to $3.3 \mathrm{pC} \mathrm{N}^{-1}$ following [29, 30] for an AT-cut quartz crystal resonator. $K=0.636 \times 10^{10} \mathrm{~N} \mathrm{~m}^{-1}$ has been estimated for the shear thickness mode of oscillation with [33]

$$
K=\frac{W L}{t} G_{\mathrm{s}} \frac{\pi^{2}}{2},
$$

where $G_{\mathrm{s}}=31 \mathrm{GPa}$ is the shear modulus of the quartz. Ignoring its convex shape, its effective dimensions are $t=$ $0.362 \mathrm{~mm}, w=1.9 \mathrm{~mm}$ and $L=7.92 \mathrm{~mm}$.

This sensor has a resonance frequency around a hundred times higher than the silicon cantilever with protruding tip used for the manipulation operation (Nanosensors ATEC-FM) [5]. This, combined with the high quality factor and stiffness, make this quartz a high speed, very sensitive sensor with amplitudes of oscillation in the sub-Ångstrom range making
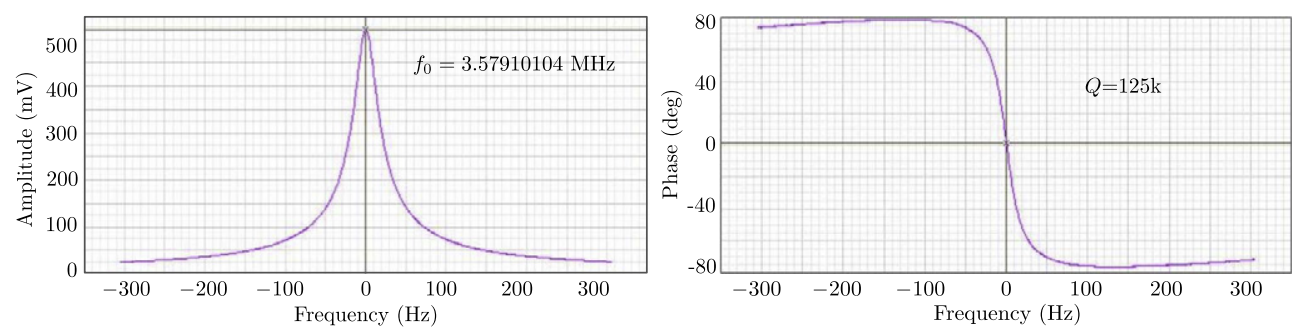

Figure 5. A $600 \mathrm{~Hz}$ frequency sweep around the resonant frequency of a quartz oscillator with a tip. (a) Amplitude and (b) phase. The resonant frequency is estimated as $f_{0}=3.57910104 \mathrm{MHz}$ with $10 \mathrm{MHz}$ precision with the OC4 controller and the quality factor $Q=125 k$. 


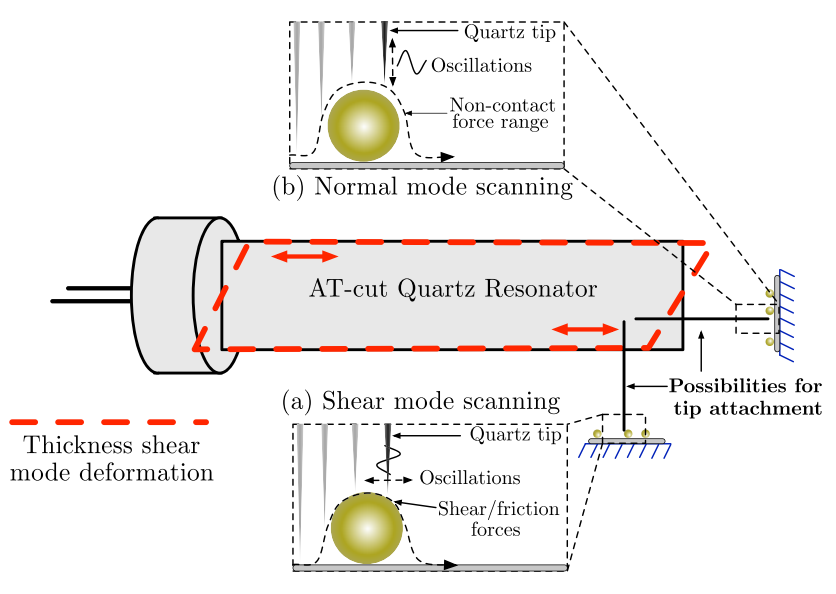

Figure 6. Tip attachment to the AT-cut quartz oscillator to obtain (a) a shear mode (friction forces) and (b) a normal mode (non-contact forces).

it ideal for fast true-non-contact-mode imaging. Additionally, the stiff probe avoids the jump-to-contact problem due to attractive surface forces and avoids bending during pull-off with liquid meniscus forces due to the presence of water in air operation. In order to take advantage of the high speed of the sensor, the AFM controller has been improved to increase the detection bandwidth compared to the previous version of the two-tip nanomanipulation AFM platform published previously in $[5,15,26]$.

\subsection{Probe dynamics and sensitivity}

The oscillation mode of rectangular AT-cut quartz resonators has been studied in several works [31-33]. It is known with precision that the AT-cut quartz resonator fundamental oscillation mode is in the thickness shear mode, as depicted in figure 6. These oscillations are parallel to the surface. The amplitude of these oscillations decreases with the distance to the center of the quartz. This has been validated experimentally with the quartz used in this paper and also described in the literature [34]. In fact, the position of the added mass (tip with glue) has a critical role in the value of the final quality factor of the sensor. As the distance of the attachment from the center of the quartz increases, the higher the quality factor is and the lower the excitation amplitude is. at the same time, larger amplitudes of oscillation are observed in the middle area but with poor quality factors. The same mode of oscillation is also present on the other side of the sensor, but mass compensation has not been attempted to symmetrize it. In summary, the tip is attached between the middle of the quartz and the end to achieve a compromise between the oscillation amplitude and the quality factor to obtain the maximum sensitivity. This position is obtained empirically and tends to be closer to the end than the middle of the sensor. This procedure of tip placement is reproducible for each monolithic resonator.

The quartz can be used as a force sensor in two different ways. The first method for force sensing with the quartz consists in using the shear forces. This is called a lateral force sensor [2]. For the shear mode, it is the friction force gradient between the tip and the surface that is probed. In this case, the tip oscillates parallel to the substrate (figure 6(a)). For the second method, the oscillation of the quartz is in the axis of the tip (figure 6(b)). For this, the tip needs to be attached parallel to the longitudinal axis of the quartz. This is called the normal mode and non-contact forces are used to control the distance between the tip and the sample.

To obtain the shear mode, the tip oscillates parallel to the substrate. This can be achieved with two different configurations, the first of which is shown in figures 6(a) and 7(a), and the second of which is shown in figure 7(b). An example of a distance spectroscopy curve is illustrated in figure 8. In this case, the frequency shift increases with friction due to the increase of contact force. It is suspected that the compression transmitted by the tip in the shear mode of oscillation of the monolithic resonator acts differently for a normal or a parallel strain. This point requires further investigation and deserves more numerical simulations taking the tip into account with regard to its influence on the oscillation modes of the quartz. The set point should be as small as possible to avoid wear of the tip and modification of the substrate.

It is challenging to obtain the normal mode due to the length of the tip that needs to be attached. Additionally, the longitudinal axis of the quartz would be aligned with the $Z$-axis, making the overall height of the probe too long compared to the focal distance of the optical microscope on top of the AFM. Thus, in order to make a probe sensitive to the non-contact forces, the tip has been attached with an angle

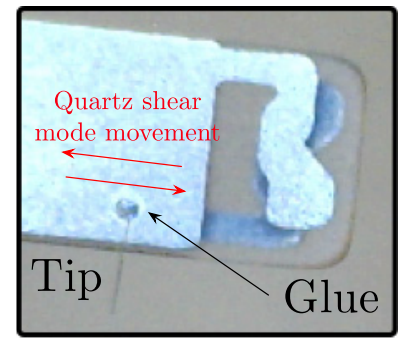

(a)

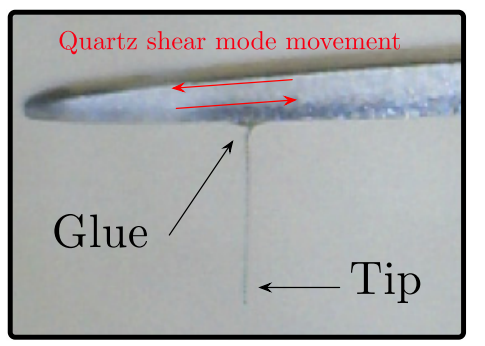

(b)

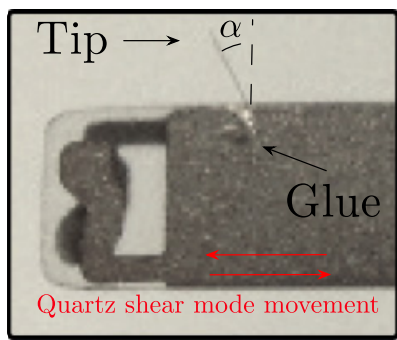

(c)

Figure 7. Tip attachment to the quartz electrode to obtain (a) a shear mode with parallel attachment, (b) a shear mode with normal attachment and (c) a hybrid mode with a parallel attachment with $\alpha=30^{\circ}$. 


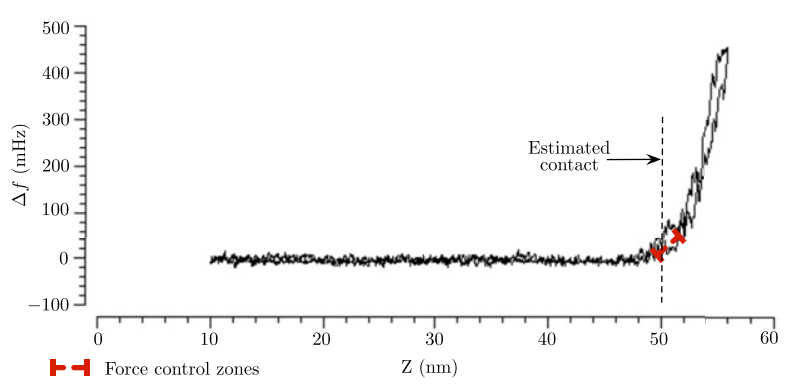

Figure 8. The approach/retract spectroscopy curve obtained with quartz in the lateral/shear mode on a silicon surface. The setpoint in the frequency shift is set in the control zone.

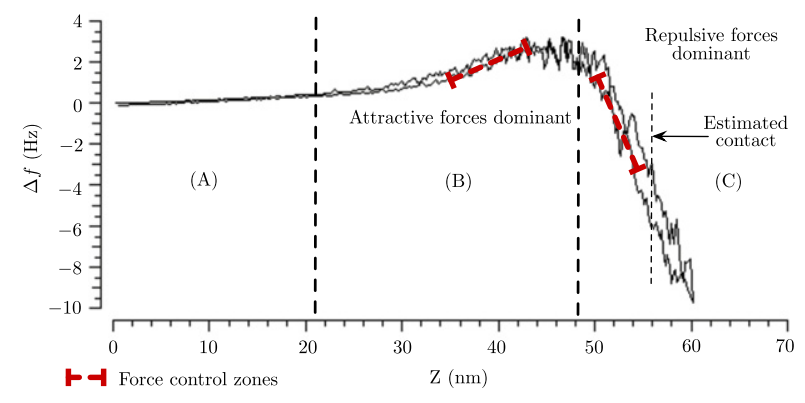

Figure 9. The approach/retract spectroscopy curve obtained with quartz in a hybrid mode on a silicon surface. (A) Before sensing any force. (B) Attractive forces are dominant compared to repulsive forces, thus $\Delta f$ is positive. (C) Repulsive forces are dominant. The setpoint in the frequency shift can be set in either the attractive or the repulsive control zone. Contact is estimated a few nanometers after $\Delta f$ decreases.

$\alpha$ (figure 7(c)). It is a hybrid configuration, and it is sensitive to both non-contact and friction forces. For this configuration, the distance spectroscopy curve is depicted in figure 9 . This mode appears very sensitive to the attractive (B) and then repulsive $(\mathrm{C})$ regimes of the surface forces. The tip diameter has been determined by SEM to be about $100 \mathrm{~nm}$, which may explain the large attractive forces observed. To control the quartz force in order to control the distance between the tip and the sample in this mode, the frequency shift setpoint can be set in either the attractive or the repulsive zone (dashed bars on top of the curve in figure 9). The attractive regime is more stable because the range is larger. In consequence, the setpoint can be set far from regions of inversion of behavior, and so inversion of the controller. The controller's parameters were set for only one of the regions; in consequence, if there is a swap from attractive to repulsive due to noise, for example, the controller will not work. Therefore, a set point close to the inversion of behavior should be avoided. In the repulsive zone there is a risk of hard contact of the tip with the substrate due to the high stiffness of the sensor.

\section{Results and discussion}

\subsection{High speed imaging with dynamic force feedback}

3.1.1. Hard surface imaging. In order to validate the speed of operation of the system and to calibrate the open

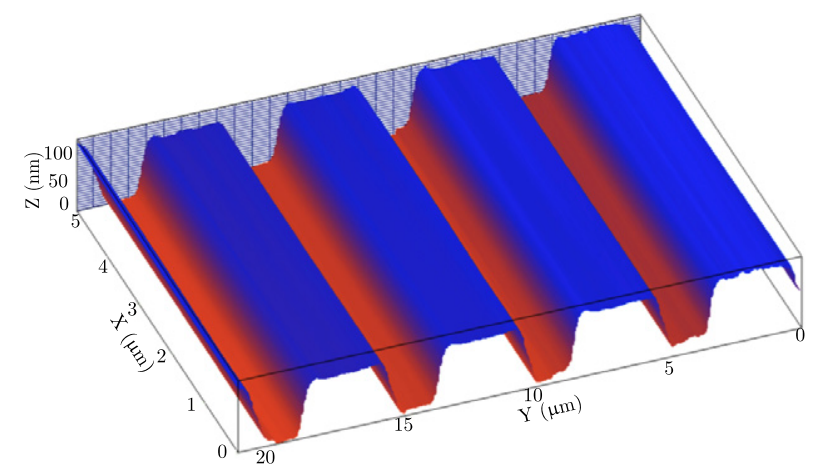

Figure 10. Raw data from the AFM scan on a hard surface with a high frequency quartz AFM probe for calibration of an open loop piezoscanner with a calibration substrate (HS-100MG from Budget Sensors). $20 \mu \mathrm{m}$ (400 pixels) in the $X$-axis and $5 \mu \mathrm{m}$ (100 pixels) in the $Y$-axis. The image acquisition time is $12.6 \mathrm{~s}$ at 32 lines ( $X$-axis) per second.

loop piezoscanner (shown in figure 2), an AFM image of a calibration grid substrate has been made (figure 10). On this AFM topography map, due to the size of the pitch of the substrate $(5 \mu \mathrm{m})$, the closed loop nanostage on the $X-Y$ axis is used to obtain a large enough lateral excursion. For the $Z$-axis the open loop piezoscanner is necessary due to the higher resonant frequency to perform faster scanning compared to the closed loop. The lateral dimensions of the image are $20 \mu \mathrm{m} \times 5 \mu \mathrm{m}$. To avoid the dependence of the $Z$-control on the image acquisition, the trenches are aligned with the fast axis of the scanning $(X)$ prior to scanning with an optical microscope. The quartz used for this example is working in a hybrid mode. The attractive forces control zone is used for controller stability by reducing the risk of contact. The $X-Y-Z$-piezo controllers' scanning speeds are manually set, resulting in an image acquisition time of $12.6 \mathrm{~s}$. This is an important improvement of fifty times in acquisition time compared to the acquisition speed of the previous version of the system [15]. The AFM software controller had to be redesigned to be able to handle the increased data flow from the higher scanning speeds. It has been developed on a real time operating system (Debian squeeze with rtai patch) and the precision has increased from $1-10 \mathrm{~ms}$ to $1-10 \mu \mathrm{s}$. In this particular case, the controller was not set at full speed, and the step time used with the real time operating system was twenty times higher than the precision/latency of the system.

3.1.2. Imaging of loose objects on a hard surface. After the experiments on hard surfaces and calibration of the piezoscanner had been carried out, gold colloid beads of different sizes (Ted Pella, Inc) were deposited on top of a silicon substrate using the method described in [35]. Initially, two images of $150 \mathrm{~nm}$ diameter beads were conducted using the hybrid force mode (figure 11(a)) and friction force mode (figure 11(b)). This is a repeatable process and both techniques are noninvasive with the substrate. The diameter of the nanospheres was estimated using the height of the sphere in the image, avoiding the lateral convolution effect of the tip end, making the dimensions seem larger in the image than 


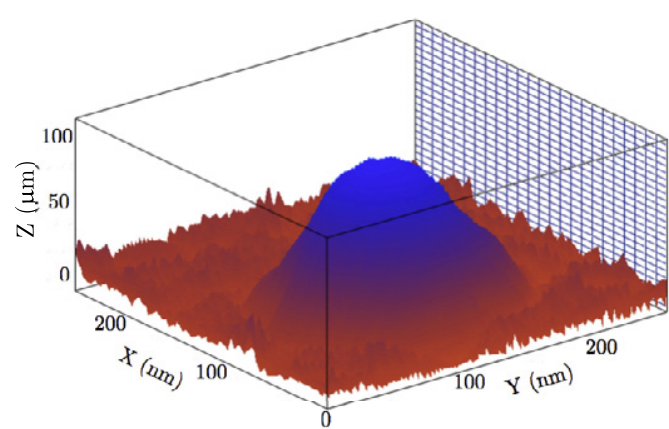

(a) Hybrid mode

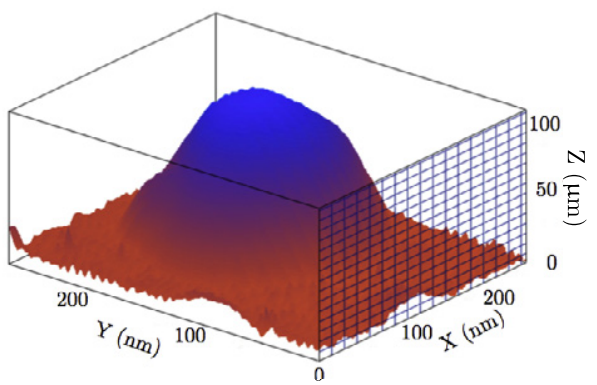

(b) Shear mode

Figure 11. A single nanosphere with (a) the hybrid mode, $250 \mathrm{~nm}$ (60 pixels) in the $X$-axis and $250 \mathrm{~nm}$ (250 pixels) in the $Y$-axis and (b) the shear mode, $250 \mathrm{~nm}$ ( 40 pixels) in the $X$-axis and $250 \mathrm{~nm}$ (200 pixels) in the $Y$-axis.

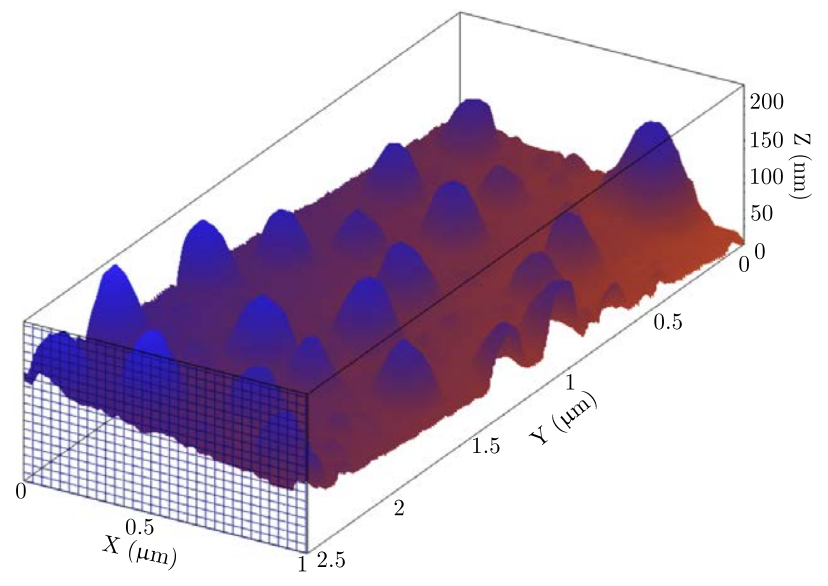

Figure 12. AFM image of gold colloid beads of $80 \mathrm{~nm}$ diameter. The image size is $1 \mu \mathrm{m}$ (40 pixels) in the $X$-axis and $2.5 \mu \mathrm{m}$ (450 pixels) in the $Y$-axis. The image acquisition time is $72 \mathrm{~s}$ at 6.43 $(X$-axis) lines per second.

they are. The image quality with both modes of operation was the same and this was mainly due to the small amplitudes of oscillation.

Under an optical microscope (Olympus BX50WI) it is easier to see the tip of the quartz when it is working in the shear mode (figure 7(a)). This is particularly interesting for the coarse approach between the two tips. Primarily for this advantage, it was selected as the imaging mode for the following experiments.

Thereafter, an AFM image in a larger range was conducted on several nanospheres of $80 \mathrm{~nm}$ diameter. The raw data of the image can be seen in figure 12. The slope of the substrate on the image is due to the alignment between the open loop piezoscanner and the substrate and not to an uncontrolled drift effect, such as thermal drift. The pyramidal shape of the imaged spheres is mainly due to the tip II apex geometry of the quartz probe in addition to the attachment angle of the tip. This scan was repeated several times without modification of the positions of the nanospheres. The scanning speed of the tip was manually set to obtain a relatively long acquisition time of $72 \mathrm{~s}$, mainly to avoid physical damage to the tip. This experiment validated the new AFM imaging arm of the two-tip AFM system with the high frequency quartz probe, in terms of the data acquisition speed and processing for the electronics. In addition, both normal and lateral modes have been experimentally validated. In section 3.2, an application in nanomanipulation of the new version of the system is shown. The high frequency quartz tip is used for fast imaging in combination with the cantilever tip for manipulation.

\subsection{Combined high speed imaging and nanomanipulation}

3.2.1. Overview of the manipulation task time. To depict the advantages of the parallel imaging and manipulation task, the whole manipulation task time needs to be analyzed. The task time, $t_{\text {task }}$, of the parallel imaging/manipulation operation can be given by [15]

$$
t_{\mathrm{task}}=\max \left(t_{\mathrm{s}}, t_{\mathrm{m}}\right)+t_{\mathrm{s}}
$$

where $t_{\mathrm{s}}$ is the scanning time of one image frame and $t_{\mathrm{m}}$ is the total manipulation time estimated from the sum of the manipulation time of each single nanoobject. For the normal-speed AFM, $t_{\text {task }}$ is often equal to $2 \times t_{\mathrm{s}}$, except for a complex manipulation task that cannot be fulfilled within one frame period, where the manipulation task time is $t_{\mathrm{m}}+t_{\mathrm{s}}$. Even if the duration is reduced from $2 \times t_{\mathrm{s}}$ to $t_{\mathrm{m}}+t_{\mathrm{s}}$, at the best, it cannot be improved further. Hence, most improvement in efficiency lies in reducing $t_{\mathrm{s}}$. The novel two-tool system presented here targets this aim while avoiding any compromises in manipulation dexterity. Improvement of the manipulation speed $t_{\mathrm{m}}$ is not within the scope of this work; in consequence the manipulation process was carried out serially with the imaging.

3.2.2. Manipulation protocol. The coordination of the two arms of the system requires a careful planning of the manipulation operation. The following steps detail the progress of a complete task.

(i) The first step is the initial approach of both tips to the substrate. For tip I, this is carried out with successive movement steps between the motorized microstage and the closed loop nanostage in the $Z$-axis until the contact is in the motion range of the nanostage. For tip II, the approach is carried out with the manual microstage and 


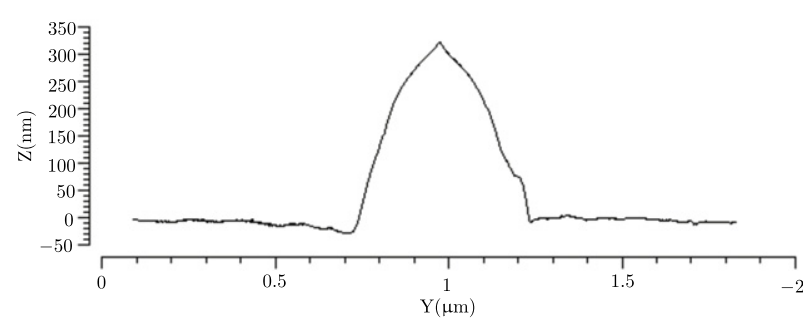

Figure 13. Scan in the $Y$-axis with tip II to find tip I.

the open loop piezostage. Additionally, with the aid of the optical microscope, the two tips are brought closer in the $X-Y$ plane until tip I is within range of tip II $(<10 \mu \mathrm{m})$.

(ii) Once the tips and the substrate are within range of the piezoactuators, $Y$-axis scans are carried out with yip II in order to find tip I (figure 13).

(iii) Now that the position of tip I is known with respect to tip II, an AFM image of the substrate is scanned with tip II. The end of tip I should appear in the image as well as the objects to manipulate. This allows the positions of objects with respect to tip I to be known. The open loop piezo is used to scan the surface with tip II (quartz) to avoid changing/modifying the distance between the other tip (tip 1, cantilever) and the surface. In this way, the edge of tip I can be scanned by tip II and be seen in the AFM image.

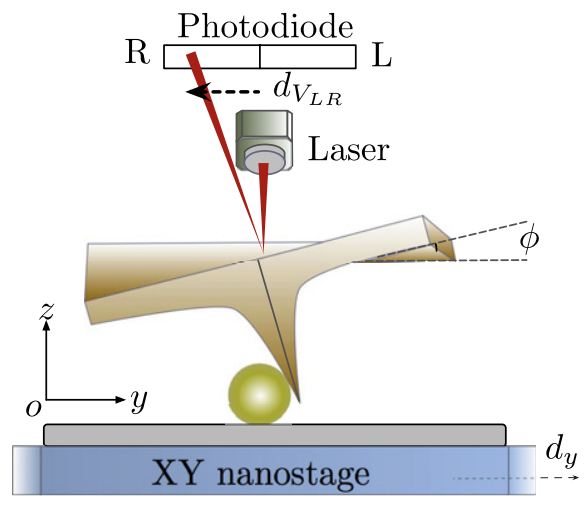

(a) (iv) The next step is the 2D manipulation of nanoobjects on the substrate (figure 14). The cantilever of tip I is used in the static mode. The force is monitored during the entire process to detect the contact between tip I and the object as well as the pushing. The closed loop nanostage is used to move the substrate.

(v) Finally, tip II is used to scan the surface again in order to obtain an AFM image of the substrate with the new positions of the objects.

3.2.3. Manipulation results. The result from a manipulation task of three gold colloid nanobeads of 80-150 nm diameter following the protocol from section 3.2.2 is presented here. This result demonstrates the nanomanipulation efficiency of the two-tip AFM to operate in ambient conditions. Once the two tips are within range according to the first step, the edge of tip I in contact with the surface is found while scanning with tip II, as seen in the profile of figure 13. Hence, the relative position between each tip is estimated. The triangular shape in the top of the scan in addition to the measured height allows differentiation from a nanosphere.

Afterwards, an AFM image is made with tip II starting near tip I and getting further away, as shown in figure 15(a). Once the position of the tip with respect the nanospheres is known, the nanoparticles are moved using tip I. In this particular case, three nanospheres were pushed to show the manipulation capabilities of the new system with two cooperative scanning probe arms.

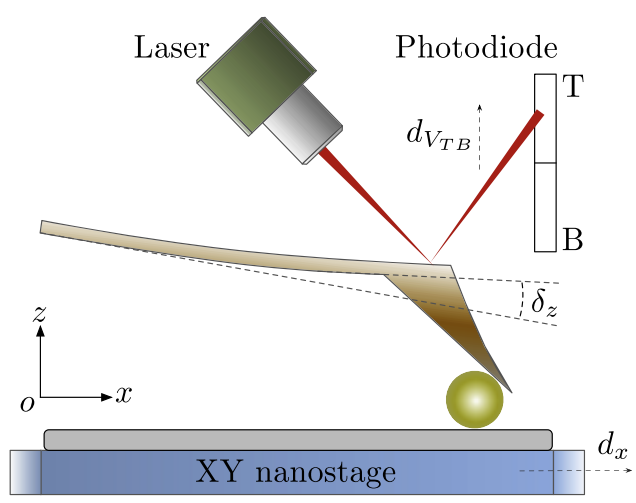

(b)

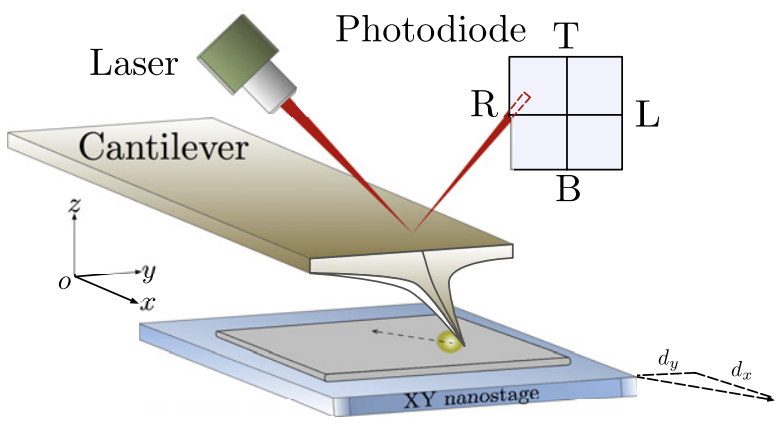

(c)

Figure 14. Diagonal pushing of a nanosphere. (a) Lateral bending, (b) $Z$-axis bending, (c) diagonal bending of the cantilever. 

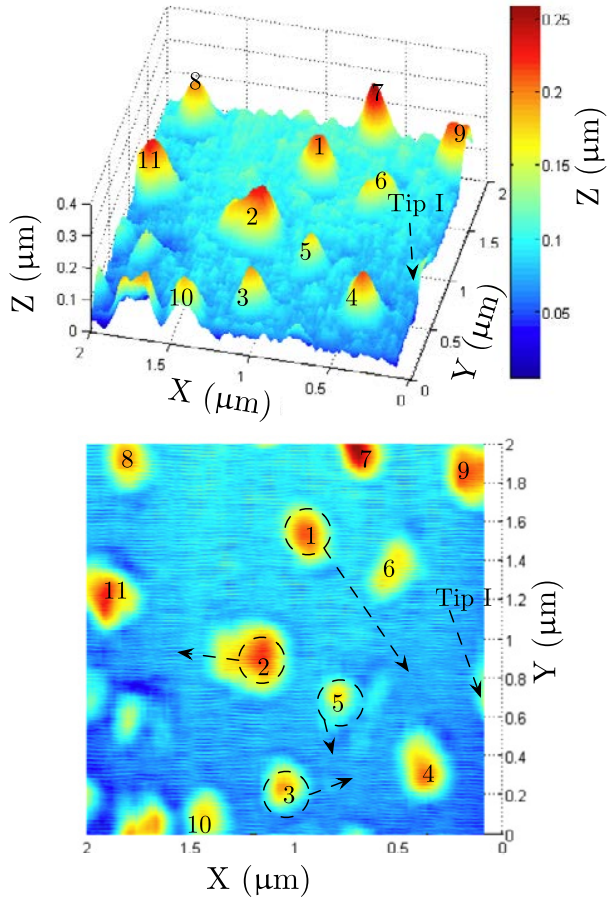

(a)
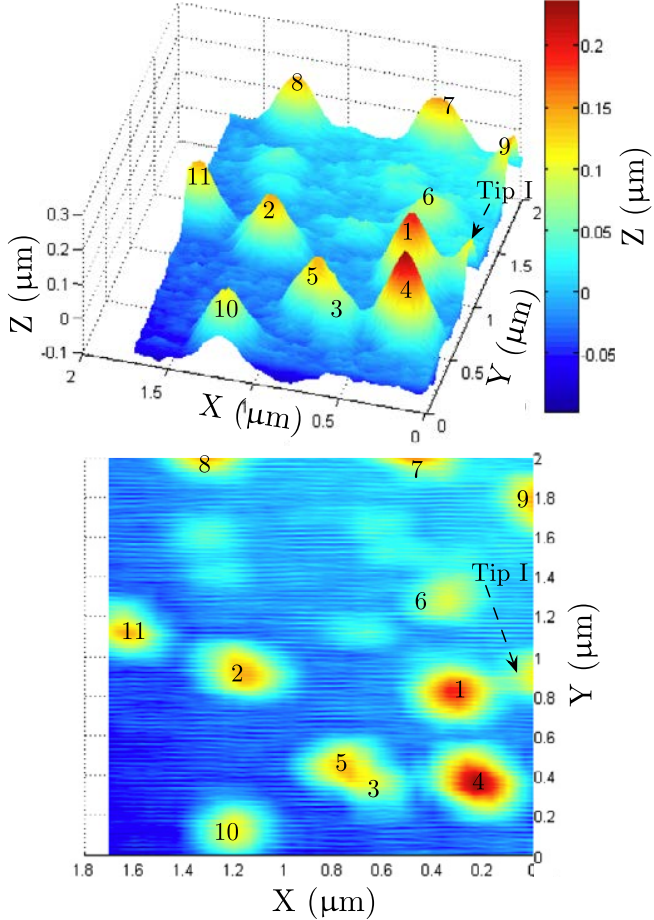

(b)

Figure 15. (a) An image with tip II before manipulation. The image size is $2 \mu \mathrm{m}$ (150 pixels) in the $X$-axis and $2 \mu \mathrm{m}$ (750 pixels) in the $Y$-axis. The image acquisition time is $108 \mathrm{~s}$ at 1.4 ( $X$-axis) lines per second. (b) An image with tip II after manipulation. The image size is 2 $\mu \mathrm{m}$ (60 pixels) in the $X$-axis and $2 \mu \mathrm{m}$ (600 pixels) in the $Y$-axis The image acquisition time is $79 \mathrm{~s}$ at 0.76 ( $X$-axis) lines per second.

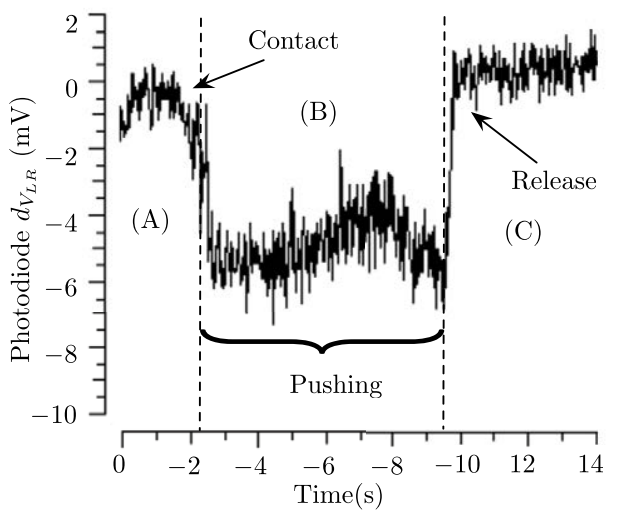

(a)

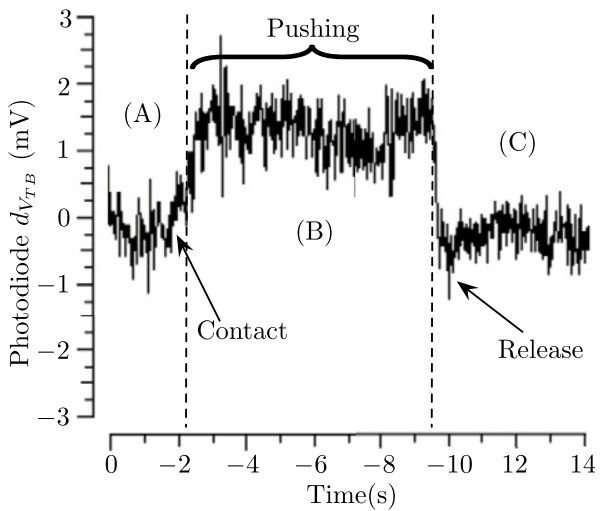

(b)

Figure 16. Photodiode voltage during diagonal pushing of a nanosphere with tip I. (a) Lateral bending and (b) $Z$-axis bending.

During the pushing of the nanospheres, the lateral and normal force behaviors are monitored with tip I, and they are estimated from the photodiode output and cantilever stiffness. An example of the output of the photodiode for diagonal pushing (figure 14(c)) of gold colloid bead number 1 can be seen in figure 16. As a result of the diagonal pushing, forces in both the $X$ - and $Y$-axes are felt. The pushing in the $X$-axis makes the cantilever bend up, resulting in a positive voltage shift (figure 16(b)). In the $Y$-axis, the cantilever bends counter-clockwise, resulting in a negative voltage shift (figure 16(a)). The behavior between the bending of the cantilever and the sign of the voltage shift is predefined by the geometrical configuration of the laser, cantilever and photodiode.

The final manipulation result is depicted in figure 15(b). Nanospheres 1-3 and 5 with diameters of 120, 90, 150 and $90 \mathrm{~nm}$ were successfully pushed with force feedback to the desired position. In this last image, the diameter of the colloids increased in the $X-Y$-axis due to the wear of the tip; this is a consequence of the large diameter apex of the tip used combined with the shear mode. The image size of 15 (a) before manipulation is $2 \mu \mathrm{m}$ (150 pixels) in the $X$-axis and $2 \mu \mathrm{m}$ (750 pixels) in the $Y$-axis. The image acquisition time is $108 \mathrm{~s}$ at 1.4 ( $X$-axis) lines per second. The image size of 
15(b) after the displacement of the three nanobeads is $2 \mu \mathrm{m}$ (60 pixels) in the $X$-axis and $2 \mu \mathrm{m}$ (600 pixels) in the $Y$-axis. The image acquisition time is $79 \mathrm{~s}$ at 0.76 ( $X$-axis) lines per second. Due to the slow response of the $Z$ piezoscanner, the $X-Y$ piezoscanner speed was tuned down. The high speed piezoscanner control was not within the scope of this work, although the literature already contains new insights on this topic [19]. The pushing or pulling of the nanospheres was not automated and the process was not within the scope of this work. In consequence, manipulation times were not considered, but only image acquisition times.

\section{Conclusion}

A dual-tip nanomanipulation AFM platform working in ambient conditions equipped with a high speed piezoelectric self-sensing scanning probe for fast AFM imaging and a cantilever for manipulation is demonstrated. The quartz oscillator used as a self-sensing scanning probe provides a more compact integration compared to an external optical lever, avoiding in consequence artifact effects of optics like interference noise and different frames of reference for the moving cantilever and fixed laser/photodiode scheme. The sub-picometer amplitude oscillation combined with the high resonant frequency $(\mathrm{MHz})$ make this dynamic force sensor ideal for fast AFM imaging. Even though the system is limited by the response of the piezoscanner actuators, the speed of the manipulation process has increased by at least five times. The needle-like configuration of the quartz with a millimeter long tip allowed easier placement on the sample compared to the micrometer long tip of a standard cantilever.

In our next experiments, integration of high speed piezoscanner control $[19,20]$ with high speed dynamic force feedback is planned, as this is the speed bottleneck of the system described in this paper. Evolution to a complete self-sensing nanomanipulation platform with a piezoresistive microlever for the manipulation operation instead of the optical cantilever is the next step. Therefore, not only will the manipulation and imaging time be reduced, but also a fully dynamic system will decrease the high coordination times between the arms prior to the manipulation. Furthermore, nanomanipulation in liquid environments for biology could be achieved, as piezoelectric self-sensing to explore proteins has recently been demonstrated [21, 36]. Further studies on modeling of the high frequency quartz oscillation mode with a tip are required in order to estimate quantitatively the force applied during the imaging and manipulation procedures.

Force feedback has been a major characteristic of atomic force microscopes for breaking micro- and nanomanipulation barriers. High speed dynamic self-sensing force feedback is a new technique for improving the performance of these systems and could be used to achieve high speed coordination of more than two arms for parallel imaging and 3D manipulation or parallel manipulation. This advance suggests a serious prospect for fully automated micro/nanoassembly and characterization of NEMSs and MEMSs.

\section{Acknowledgments}

We would like to thank Gilgueng Hwang and Bruno Sauvet for their fruitful discussions. This work has been supported by the French National Project NANOROBUST ANR-2011 NANO 00601.

\section{References}

[1] Fatikow S (ed) 2007 Automated Nanohandling by Microrobots (Berlin: Springer)

[2] Giessibl F J 2003 Advances in atomic force microscopy Rev. Mod. Phys. 75949

[3] Gauthier M and Régnier S (ed) 2010 Robotic Micro-Assembly (New York: Wiley-IEEE Press)

[4] Nanonics Imaging Ltd www.nanonics.co.il/applications/afm_ sem_integration

[5] Xie H and Régnier S 2011 Development of a flexible robotic system for multiscale applications of micro/nanoscale manipulation and assembly IEEE/ASME Trans. Mech. $16266-76$

[6] Millet O, Bernardoni P, Régnier S, Bidaud P, Tsitsiris E, Collard D and Buchaillot L 2004 Electrostatic actuated micro gripper using an amplification mechanism Sensors Actuators A 114 371-8

[7] Pérez R, Agnus J, Clévy C, Hubert A and Chaillet N 2005 Modeling, fabrication, and validation of a high-performance 2-DoF piezoactuator for micromanipulation IEEE/ASME Trans. Mechatronics 10 161-71

[8] Driesen W, Varidel T, Régnier S and Breguet J M 2005 Micromanipulation by adhesion with two collaborating mobile micro robots J. Micromech. Microeng. 15 S259-67

[9] Kim P and Lieber C M 1999 Nanotube nanotweezers Science 286 2148-50

[10] Requicha A A 2003 Nanorobots, NEMS, and nanoassembly Proc. IEEE 91 1922-33

[11] Sitti M and Hashimoto H 2000 Controlled pushing of nanoparticles: modeling and experiments IEEE/ASME Trans. Mechatronics 5 199-211

[12] Guthold M, Falvo M R, Matthews W G, Paulson S, Washburn S, Erie D A, Superfine R, Brooks F P and Taylor R M 2000 Controlled manipulation of molecular samples with the nanomanipulator IEEE/ASME Trans. Mechatronics 5 189-98

[13] Resch R, Lewis D, Meltzer S, Montoya N, Koel B E, Madhukar A, Requicha A A G and Will P 2000 Manipulation of gold nanoparticles in liquid environments using scanning force microscopy Ultramicroscopy 82 135-9

[14] Sitti M 2004 Atomic force microscope probe based controlled pushing for nanotribological characterization IEEE/ASME Trans. Mechatronics 9 343-9

[15] Xie H, Haliyo D S and Régnier S 2009 Parallel imaging/manipulation force microscopy Appl. Phys. Lett. 94153106

[16] Fantner G E, Hegarty P, Kindt J H, Schitter G, Cidade G A G and Hansma P K 2005 Data acquisition system for high speed atomic force microscopy Rev. Sci. Instrum. 76026118

[17] Hansma P K, Schitter G, Fantner G E and Prater C 2006 High-speed atomic force microscopy Science $\mathbf{3 1 4} 601$

[18] Seo Y, Choi C S, Han S H and Han S J 2008 Real-time atomic force microscopy using mechanical resonator type scanner Rev. Sci. Instrum. 79103703

[19] Ando T, Uchihashi T and Fukuma T 2008 High-speed atomic force microscopy for nano-visualization of dynamic biomolecular processes Prog. Surf. Sci. 83 337-437 
[20] Picco L M, Bozec L, Ulcinas A, Engledew D J, Antognozzi M, Horton M A and Miles M J 2007 Breaking the speed limit with atomic force microscopy Nanotechnology 18044030

[21] Makky A, Berthelot Th, Feraudet-Tarisse C, Volland H, Viel P and Polesel-Maris J 2012 Substructures high resolution imaging of individual $\mathrm{IgG}$ and $\mathrm{IgM}$ antibodies with piezoelectric tuning fork atomic force microscopy Sensors Actuators B 162 269-77

[22] Karrai K and Grober R D 1995 Piezoelectric tip-sample distance control for near field optical microscopes Appl. Phys. Lett. 661842

[23] Acosta J C, Hwang G, Polesel-Maris J and Régnier S 2011 A tuning fork based wide range mechanical characterization tool with nanorobotic manipulators inside a scanning electron microscope Rev. Sci. Instrum. 82035116

[24] Albrecht T R, Grütter P, Horne D and Rugar D 1991 Frequency modulation detection using high-Q cantilevers for enhanced force microscope sensitivity J. Appl. Phys. $69668-73$

[25] EPSON TOYOCON data sheets on: www.epsontoyocom.co. jp/english/product/Crystal/index.html

[26] Xie H, Haliyo D S and Régnier S 2009 A versatile atomic force microscope for three-dimensional nanomanipulation and nanoassembly Nanotechnology 21215301

[27] Krejci P and Kuhnen K 2001 Inverse control of systems with hysteresis and creep IEEE Proc. Control Theory Appl. 148 185-92

[28] Abe T, Shimamoto H and Li X 2006 Miniaturization of spherically contoured rectangular AT-cut quartz-crystal resonators by using reactive ion etching Japan. J. Appl. Phys. 45 5283-5
[29] Yongho S, Hwansung C and Wonho J 2003 Atomic-resolution noncontact atomic force microscopy in air Appl. Phys. Lett. 831860

[30] Yongho S and Wonho J 2005 Tapping mode quartz crystal resonator based scanning force microscopy Rev. Sci. Instrum. 76016106

[31] Jeong H-W, Aoki T and Hatsuzawa T 2004 Frequency responses of spherically contoured rectangular AT-cut quartz crystal resonators fabricated by fixed abrasive method Int. J. Mach. Tools Manuf. 44 1143-9

[32] Sekimoto H, Tajima D, Watanabe Y and Ishizaki A 1995 Application of Lee's plate equations to analysis of spurious vibrations of rectangular AT-cut quartz plates Japan. J. Appl. Phys. 34 5706-10

[33] Johannsmann D 2008 Viscoelastic, mechanical, and dielectric measurements on complex samples with the quartz crystal microbalance Phys. Chem. Chem. Phys. 10 4516-34

[34] Sun H, Lu P, Zhang P and Chen H 2004 Dynamic analysis of AT-cut quartz resonators with ANSYS Sensors Proc. IEEE $195-8$

[35] Lee K, Duchamp M, Kulik G, Magrez A, Seo J W, Jeney S, Kulik A J, Forró L, Sundaram R S and Brugger J 2007 Uniformly dispersed deposition of colloidal nanoparticles and nanowires by boiling Appl. Phys. Lett. 91173112

[36] Polesel-Maris J, Legrand J, Berthelot Th, Garcia A, Viel P, Makky A and Palacin S 2011 Force spectroscopy by dynamic atomic force microscopy on bovine serum albumin proteins changing the tip hydrophobicity, with piezoelectric tuning fork self-sensing scanning probe Sensors Actuators B $161775-83$ 